\title{
Prediction Ability of Charlson, Elixhauser, and Rx-Risk Comorbidity Indices for Mortality in Patients with Hip Fracture. A Danish Population-Based Cohort Study from 20I4-2018
}

\author{
Jeppe Damgren Vesterager (D, Morten Madsen (D), Thomas Johannesson Hjelholt (D), \\ Pia Kjær Kristensen (D, Alma Becic Pedersen (D) \\ Department of Clinical Epidemiology, Aarhus University Hospital and Department of Clinical Medicine, Aarhus University, Aarhus, Denmark \\ Correspondence: Alma Becic Pedersen, Tel +45 87/672I2, Fax+45 87I672I5, Email abp@clin.au.dk
}

Objective: Comorbidity has an important role in risk prediction and risk adjustment modelling in observational studies. However, it is unknown which comorbidity index is most accurate to predict mortality in hip fracture patients. We aimed to evaluate the prediction ability, including discrimination and calibration of Charlson comorbidity index (CCI), Elixhauser comorbidity index (ECI) and Rx-risk index for 30 day- and 1 year mortality in a population-based cohort of hip fracture surgery patients.

Methods: Using the Danish Multidisciplinary Hip Fracture Registry in the period 2014-2018, 31,443 patients were included. CCI and ECI were based on discharge diagnoses, while Rx-Risk index was based on pharmacy dispensings. We used logistic regression to assess discrimination of the different indices, individually and in combinations, by calculating c-statistics and the contrast in c-statistic to a base model including only age and gender with $95 \%$ confidence intervals $(\mathrm{CI})$.

Results: The study cohort were primarily female (69\%) and older than 85 years (42\%). The 30-day mortality was $10.1 \%$ and the 1 -year mortality was $26.6 \%$. Age and gender alone had a good discrimination ability for 30-day and 1-year mortality (c-statistic=0.70, CI: $0.69-$ 0.71 and c-statistic $=0.68, \mathrm{CI}: 0.67-0.69$, respectively). By adding indices individually to the base model, Rx-risk index had the best 30 -day and 1-year mortality discrimination ability (c-statistic $=0.73$, CI: $0.72-0.74$ and 0.71 CI: $0.71-0.72$, respectively). By adding combination of indices to the base model, a combination of CCI and the Rx-risk index had a 30-day and 1-year mortality discrimination ability of c-statistic $=0.74$, CI: $0.73-0.75$ and c-statistic $=0.73$, CI: $0.73-0.74$, respectively. Calibration of indices was similar.

Conclusion: The highest discrimination ability was achieved by combining CCI and Rx-risk index in addition to age and gender. However, age and gender alone had a fair mortality discrimination ability.

Keywords: Charlson comorbidity index, elixhauser index, Rx-risk index, multimorbidity, discrimination, calibration

\section{Introduction}

Hip fracture is a common condition among elderly. ${ }^{1}$ Patients are at high risk of developing postoperative complications, increased disability, and have a 1-year mortality of approximately $30 \% .{ }^{1-3}$ Furthermore, comorbidities are common among hip fracture patients, with more than $75 \%$ of patients having a chronic disease. Patients with higher comorbidity burden are at higher risk of mortality and postoperative complications. ${ }^{3,4}$ In observational studies, comorbidity indices are widely used for confounder adjustment among hip fracture patients. Two frequently used diagnosis-based comorbidity indices are the Charlson comorbidity index ${ }^{5-8}(\mathrm{CCI})$ and the Elixhauser comorbidity index (ECI). ${ }^{7-10}$ Diagnosis-based comorbidity indices have the disadvantage of being subject to variations in coding of diagnoses both between clinicians and between Hospitals. As well as, a lack of diagnoses from outpatient clinics and general practices. ${ }^{11}$ Therefore, prescription-based comorbidity indices has been developed ${ }^{12}$ such as the Rx-risk index. Rx-risk index could capture both severe but also less severe conditions not requiring hospital contact and diagnosis. 
One previous study has investigated the prediction ability in terms of discrimination of CCI and ECI for mortality in hip fracture patients. However, this study only included patients operated with arthroplasty ${ }^{8}$ for a femoral neck fracture, not including patients with a sub- or per-trochanteric fracture, which account for about $50 \%$ of all hip fracture patients. ${ }^{13}$ Nevertheless, the study concluded that a model with age and gender alone had superior discrimination of mortality compared with complex $\mathrm{CCI}$ and $\mathrm{ECI} .{ }^{8}$ The Rx-risk index has not previously been investigated in discriminating mortality among hip fracture patients. Thus, it remains unclear which comorbidity index is most accurate in predicting the mortality of hip fracture patients. Therefore, it is unknown which comorbidity index is best for confounder adjustment in epidemiological observational studies based on registry data. Better control for comorbidity confounding might lead to increase in internal validity of future epidemiological studies of hip fracture patients and improve basis for clinical decision making. We hypothesized that more complex indices may improve on the capture of patient's preoperative comorbidity and prediction of mortality compared to a simple measure based on a few variables such as age and gender.

The aim of this study was to compare predictive ability of CCI, ECI and Rx-risk index individually and in combination for 30 day- and 1 year mortality in a hip fracture surgery population. We focused on two traditional measures of the quality of prediction, thus discrimination and calibration of indices. ${ }^{14}$

\section{Method}

\section{Study Design, Setting and Participants}

Our cohort study was conducted using prospectively collected data from Danish medical registries, encompassing the entire Danish population, which has a tax-supported health care system with free access to care. ${ }^{15}$ All patients $\geq 65$ years admitted to a hospital with a first-time femoral neck (S720), pertrochanteric, (S721), or subtrochanteric (S722) femoral fracture treated surgically with osteosynthesis or total/hemi arthroplasty from 2014 to 2018 were included from the population based clinical quality database; the Danish Multidisciplinary Hip Fracture Registry (DMHFR). ${ }^{13}$

\section{Comorbidity Measures and Data Sources}

CCI consists of a weighted score based on 19 conditions and was developed using information from 559 breast cancer patients. ${ }^{5}$ The ECI is a comorbidity measure developed with the purpose to use in large administrative inpatient dataset. The ECI includes a comprehensive list of 21 comorbid conditions and is summarized by a weighted score for each comorbid condition. ${ }^{9}$ In the current study, the CCI and ECI were defined using International Classification of Diseases ICD-10 diagnosis codes from the Danish National Patient Registry (DNPR) covering all Danish hospitals. ${ }^{16}$ Primary and secondary discharge diagnoses from in-hospital and outpatient clinics with a 10-year lookback period, and with a discharge prior to hip fracture admission were included. Diagnoses recorded during the hip fracture admission were excluded due to the possibility of being complications to hip fracture treatment.

The Rx-risk index was initially developed to measure healthcare expenses, and contains 29 categories of comorbidity based on Anatomical Therapeutic Chemical Classification System codes (ATC), summarized by a weighted score for each comorbid condition. ${ }^{17}$ In the current study, the Rx-risk index was defined by ATC codes from The Danish National Prescription Registry with a one year lookback period. ${ }^{18}$

The Danish Civil Registration System (DCRS) assigns all Danish residents with a unique identification number. It is recorded at all contacts with the healthcare system and is included in all Danish registries. Therefore, an unambiguous linkage between all medical registries is possible ${ }^{15}$.

A full list of diagnosis and ATC codes is found in Supplementary Tables 1- $\underline{3}$.

\section{Outcome}

The outcome was death within 30 days and 1 year. Follow-up started on the day of surgery.

\section{Statistical methods}

The following comorbidity indices prediction ability were compared to age and gender model (base model): CCI, ECI and Rx-risk index. All comorbidity indices were investigated both as continuous variables and in categories in terms of 
predictive ability for mortality using logistic regression, assuming each observation was independent. The additional predictive ability of pairwise combinations of two distinct indices was investigated, such as the combination of CCI and Rx-risk index, the ECI and Rx-risk index, and CCI and ECI. The base model was used as reference.

For each model we assessed two traditional measures of the quality of prediction, thus, discrimination and calibration. The discrimination answers the question of how well the model can separate the persons with and without outcome (death). The discrimination should be interpreted as the probability that a randomly selected patient who experience the outcome (death) had a higher comorbidity score than a person who had not experience the outcome. Each model's discrimination ability was illustrated graphically with receiver operating characteristic (ROC)-curves. ${ }^{19}$ To construct an ROC curve, the sensitivity (true positive rate) was plotted against the 1-specificity (false positive rate) for consecutive cutoff points for the probability of death. If the model has perfect discrimination, the ROC curve should reach the upper left corner of the plot (corresponding to $100 \%$ sensitivity and $100 \%$ specificity). The area under the ROC curves is equivalent to c-statistic which we calculated with the logistic regression model. In addition, the contrasts in c-statistic to the base model as well as the respective $95 \%$ confidence intervals (CI) were calculated. The c-statistic ranges from 0 to 1 , with 1 indicating perfect discrimination and 0.5 reflecting chance. ${ }^{20}$

We assessed calibration of comorbidity models with 10-percentiles graphically, which answer the question of how well the predicted probability of death agrees with observed mortality. ${ }^{14}$

The association between the individual components included in CCI, ECI and Rx-Risk and mortality was evaluated by calculating odds ratios (OR) adjusted for age, gender and the remaining index components. Thereby, comparing patients with and without specific individual comorbidity component.

The prediction models were not tested using training and test-data, given that they were not newly developed, but already well-established prediction models.

The content of this paper follows the Strengthening the Reporting of Observational Studies in Epidemiology (STROBE) guidelines and the REporting of studies Conducted using Observational Routinely-collected Data (RECORD) guidelines. ${ }^{21}$ Analyses were performed using SAS V. 9.4 (SAS Institute Inc., Cary, NC, USA) and $\mathrm{R}$ version 3.6.1 (R Foundation for Statistical Computing).

The study was reported to the Danish Data Protection Agency through registration at Aarhus University (record number: AU-2016-051-000001, sequential number 880). Non-interventional registry-based research projects that do not involve human biological material but are based on pure data such as numbers do not require notification to the Danish Scientific Ethics Committee. ${ }^{22}$

\section{Results}

The study cohort consisted of 31,443 patients of whom the majority were female (69\%), and the most frequent age category were older than 85 years $(42 \%)$ (Table 1$)$. A total of $10.1 \%(n=3177)$ patients died within 30 days, while $26.6 \%$ $(\mathrm{n}=8366)$ patients died within 1 year. Further description of the study population and distributions of comorbidity measures in categories are provided in Table 1.

\section{0-Day Mortality}

The base model had a discrimination ability of 30-day mortality with c-statistic $=0.70, \mathrm{CI}: 0.69-0.71$. Adding CCI, ECI, Rx-Risk individually to the base model resulted in higher c-statistics of 0.72 (CI: $0.71-0.73$ ), 0.72 (CI: $0.71-0.73$ ), and 0.73 (CI: $0.72-0.74$ ), respectively. A combination of the CCI and the Rx-risk index had the best 30-day mortality discrimination ability (c-statistic $=0.74$, CI: $0.73-0.75$ ). Contrasts in c-statistics compared to the base model are provided in Table 2. Figure 1 illustrates the ROC curves for 30-day mortality for CCI, ECI, and Rx-Risk as continuous scores.

All comorbidity indices had similar discrimination ability if included as a continuous score or as in categories (Table 2). In addition, calibration of base model and all individual comorbidity indices was similarly good (Supplementary Figure 1). 
Table I Characteristics of 3I,443 Patients Undergoing Hip Fracture Surgery from 2014 to 2018

\begin{tabular}{|c|c|}
\hline & $\mathbf{N}(\%)$ \\
\hline All & $31,443(100)$ \\
\hline \multicolumn{2}{|c|}{ Age in years } \\
\hline $65-75$ & $6715(21.4)$ \\
\hline $75-85$ & II,426 (36.3) \\
\hline $85+$ & $13,302(42.3)$ \\
\hline \multicolumn{2}{|l|}{ Gender } \\
\hline Male & $9630(30.6)$ \\
\hline Female & $21,813(69.4)$ \\
\hline \multicolumn{2}{|c|}{ Body mass index } \\
\hline $10-19.9$ & $4083(13.0)$ \\
\hline $20-24.9$ & $12,450(39.6)$ \\
\hline $25-29.9$ & $6866(21.8)$ \\
\hline $30+$ & $2133(6.8)$ \\
\hline Missing & $5911(18.8)$ \\
\hline \multicolumn{2}{|l|}{ Residence } \\
\hline Own home & $21,864(69.5)$ \\
\hline Institution & $6796(21.6)$ \\
\hline Unknown & $2783(8.9)$ \\
\hline \multicolumn{2}{|c|}{ Charlson Comorbidity Index } \\
\hline 0 & I I,862 (37.7) \\
\hline I & $7301(23.2)$ \\
\hline 2 & $5653(18.0)$ \\
\hline $3+$ & $6627(21.1)$ \\
\hline \multicolumn{2}{|c|}{ Elixhauser Comorbidity Index } \\
\hline$\leq 0$ & $|3,95|(44.4)$ \\
\hline $1-5$ & 7761 (24.7) \\
\hline $6-13$ & $6800(21.6)$ \\
\hline $14+$ & $2931(9.3)$ \\
\hline \multicolumn{2}{|l|}{ Rx-Index } \\
\hline$-5-0$ & $8833(28.1)$ \\
\hline $1-3$ & $9753(31.0)$ \\
\hline
\end{tabular}

(Continued) 
Table I (Continued).

\begin{tabular}{|l|l|}
\hline & $\mathbf{N}(\%)$ \\
\hline $4-6$ & $6775(21.5)$ \\
\hline $7+$ & $6082(19.3)$ \\
\hline
\end{tabular}

\section{I-Year Mortality}

The base model had a discrimination ability of 1 -year mortality with c-statistic=0.68, CI: $0.67-0.69$. Adding CCI, ECI, Rx-Risk individually to the base model resulted in higher c-statistic of 0.71 (CI: 0.71-0.72), 0.71 (CI: 0.70-0.72), and 0.71 (CI: 0.71-0.72), respectively. A combination of the CCI and the Rx-risk index had the best 1-year mortality discrimination ability (c-statistic $=0.73, \mathrm{CI}$ : 0.73-0.740). Figure 2 illustrates the ROC curves for 1-year mortality for CCI, ECI, and Rx-Risk as continuous scores.

All comorbidity indices had similar discrimination ability if included as a continuous score and in categories (Table 2). In addition, calibration of base model and all individual comorbidity indices was similarly good (Supplementary Figure 2).

\section{Components of the Comorbidity Indices}

When looking at patients with the different components of CCI, ECI and Rx-risk index, patients having AIDS/HIV had the highest 30-day relative mortality with an adjusted OR of 3.59 (CI: 0.95-13.61) in CCI. While patients with metastatic

Table 2 C- Statistic and C-Statistic Contrast for Comorbidity Indicies Predicting 30-Day and I-Year Mortality Among 3I,443 Hip Fracture Patients

\begin{tabular}{|c|c|c|c|c|}
\hline \multirow[t]{2}{*}{ Model } & \multicolumn{2}{|c|}{30 Days Mortality } & \multicolumn{2}{|c|}{ I Year Mortality } \\
\hline & C- statistics $(95 \% \mathrm{Cl})^{\mathrm{a}}$ & $\begin{array}{l}\text { C- statistic contrast } \\
(95 \% \mathrm{Cl})^{\mathrm{b}}\end{array}$ & C- statistic $(95 \% \mathrm{Cl})^{\mathrm{a}}$ & $\begin{array}{l}\text { C- statistic contrast } \\
(95 \% \mathrm{Cl})^{\mathrm{b}}\end{array}$ \\
\hline Age and gender (Base model) & $0.699(0.690-0.709)$ & Ref. & $0.680(0.673-0.687)$ & Ref. \\
\hline Base model $+\mathrm{CCl} *$ (continuous) & $0.722(0.7|3-0.73|)$ & $0.022(0.018-0.027)$ & $0.715(0.709-0.721)$ & $0.035(0.031-0.039)$ \\
\hline Base model $+\mathrm{CCl}$ (in categories) & $0.720(0.711-0.729)$ & $0.021(0.016-0.025)$ & $0.711(0.705-0.718)$ & $0.031(0.027-0.035)$ \\
\hline $\begin{array}{l}\text { Base model + Elixhauser comorbidity index } \\
\text { (continuous) }\end{array}$ & $0.719(0.710-0.728)$ & $0.019(0.015-0.024)$ & $0.710(0.704-0.717)$ & $0.030(0.026-0.034)$ \\
\hline $\begin{array}{l}\text { Base model + Elixhauser comorbidity index } \\
\text { (in categories) }\end{array}$ & $0.718(0.709-0.727)$ & $0.019(0.014-0.023)$ & $0.710(0.704-0.716)$ & $0.030(0.026-0.034)$ \\
\hline Base model + Rx-risk index (continuous) & $0.728(0.719-0.737)$ & $0.028(0.023-0.033)$ & $0.716(0.710-0.723)$ & $0.036(0.032-0.040)$ \\
\hline Base model + Rx-risk index (in categories) & $0.726(0.717-0.735)$ & $0.026(0.02 I-0.03 I)$ & $0.714(0.708-0.72 I)$ & $0.034(0.030-0.038)$ \\
\hline $\begin{array}{l}\text { Base model }+\mathrm{CCl} \text { and Elixhauser } \\
\text { comorbidity index }\end{array}$ & $0.725(0.716-0.733)$ & $0.025(0.020-0.030)$ & $0.719(0.713-0.726)$ & $0.039(0.035-0.044)$ \\
\hline Base model $+\mathrm{CCl}$ and $\mathrm{Rx}$-risk index & $0.738(0.730-0.747)$ & $0.039(0.033-0.045)$ & $0.734(0.728-0.740)$ & $0.054(0.049-0.059)$ \\
\hline $\begin{array}{l}\text { Base model + Elixhauser and Rx-risk index } \\
\text { comorbidity index }\end{array}$ & $0.737(0.728-0.746)$ & $0.038(0.032-0.043)$ & $0.731(0.725-0.737)$ & 0.05 I $(0.047-0.056)$ \\
\hline
\end{tabular}

Notes: *Charlson Comorbidity index. ${ }^{\mathrm{a} C a l c u l a t e d}$ using the logistic regression model. ${ }^{\mathrm{b}} \mathrm{Calculated}$ as the contrast from the base model (reference group). 
Table 3 Odds Ratio (OR) for 30-Day Mortality Among 31,443 Hip Fracture Patients. Comparing Patients with and without Specific Components of Charlson Comorbidity Index, Elixhauser Comorbidity Index and Rx-Risk Index. Adjusted for Age, Gender, and the Remaining Index Components of the Index of Interest

\begin{tabular}{|c|c|c|c|c|}
\hline \multicolumn{2}{|l|}{ Component } & \multirow{2}{*}{$\begin{array}{l}\text { Charlson Comorbidity } \\
\text { Index }\end{array}$} & \multirow{2}{*}{$\begin{array}{l}\text { Elixhauser Comorbidity } \\
\text { Index }\end{array}$} & \multirow{2}{*}{$\begin{array}{l}\text { Rx-Risk Index } \\
0.43(0.16,1.18)\end{array}$} \\
\hline Addiction & Alcohol dependency/abuse & & & \\
\hline & Drug abuse & & $0.81(0.36,1.80)$ & \\
\hline & Smoking cessation & & & $1.70(1.14,2.53)$ \\
\hline \multirow[t]{6}{*}{ Cancer } & Leukemia & $2.02(1.36,3.01)$ & & \\
\hline & Lymphoma & $1.28(0.91,1.79)$ & $1.24(0.89,1.73)$ & \\
\hline & Malignancies & & & $0.45(0.10,1.96)$ \\
\hline & Metastatic solid tumor & $2.46(1.90,3.20)$ & $2.35(1.80,3.06)$ & \\
\hline & Tumor (without metastasis) & $1.10(1.00,1.22)$ & $1.13(1.01,1.26)$ & \\
\hline & Weight loss & & $1.59(1.31,1.93)$ & \\
\hline \multirow[t]{16}{*}{ Cardiovascular } & Anemia, blood loss & & $1.25(0.87,1.79)$ & \\
\hline & Anemia, deficiency & & $1.26(1.05,1.5 \mathrm{I})$ & \\
\hline & Anticoagulants & & & $1.03(0.92,1.15)$ \\
\hline & Antiplatelets & & & $1.22(1.12,1.33)$ \\
\hline & Arrhythmia & & $1.10(1.00,1.20)$ & $1.25(1.08,1.45)$ \\
\hline & Cerebrovascular disease & $1.09(0.99,1.20)$ & & \\
\hline & Coagulopathy & & $0.95(0.65,1.38)$ & \\
\hline & Congestive heart failure & $1.49(1.32,1.67)$ & $1.37(1.22,1.55)$ & $\mathrm{I} .57(\mathrm{I} .4 \mathrm{I}, \mathrm{I} .75)$ \\
\hline & Fluid and electrolyte disorders & & $1.32(1.20,1.46)$ & \\
\hline & Hyperlipidemia & & & $0.78(0.71,0.86)$ \\
\hline & Hypertension & & $0.92(0.84,1.00)$ & $1.24(1.14,1.34)$ \\
\hline & Ischemic heart disease angina & & & $1.26(1.10,1.44)$ \\
\hline & Ischemic heart disease hypertension & & & $0.97(0.90,1.05)$ \\
\hline & Myocardial infarction & $1.14(0.97,1.33)$ & & \\
\hline & Peripheral vascular disease & $1.30(1.15,1.47)$ & $1.28(1.13,1.45)$ & \\
\hline & Valvular disease & & $1.31(1.15,1.49)$ & \\
\hline \multirow[t]{7}{*}{ Endocrinology } & Diabetes, uncomplicated & $1.14(1.00,1.30)$ & $1.10(0.95,1.27)$ & \multirow[t]{2}{*}{$1.21(1.07,1.37)$} \\
\hline & Diabetes, complicated & $\mathrm{I} .02(0.74, \mathrm{I} .4 \mathrm{I})$ & $1.13(0.93,1.36)$ & \\
\hline & Hyperthyroidism & & & $1.38(0.70,2.72)$ \\
\hline & Hypothyroidism & & $0.91(0.73,1.13)$ & $0.86(0.74,1.00)$ \\
\hline & Obesity & & $\mathrm{I} .00(0.76,1.3 \mathrm{I})$ & \\
\hline & Osteoporosis/Paget's & & & $0.85(0.75,0.97)$ \\
\hline & Steroid-responsive disease & & & $1.4 \mid(1.24,1.59)$ \\
\hline
\end{tabular}

(Continued) 
Table 3 (Continued).

\begin{tabular}{|c|c|c|c|c|}
\hline \multicolumn{2}{|l|}{ Component } & \multirow{2}{*}{$\begin{array}{l}\text { Charlson Comorbidity } \\
\text { Index }\end{array}$} & \multirow{2}{*}{$\begin{array}{l}\text { Elixhauser Comorbidity } \\
\text { Index }\end{array}$} & \multirow{2}{*}{$\begin{array}{l}\text { Rx-Risk Index } \\
\text { I.I5 (I.05, I.25) }\end{array}$} \\
\hline Gastrointestinal & $\begin{array}{l}\text { Gastroesophageal reflux disease/peptic } \\
\text { ulcer }\end{array}$ & & & \\
\hline & Irritable bowel syndrome & & & $1.08(0.77,1.53)$ \\
\hline & Mild liver disease & $1.65(1.15,2.37)$ & $1.43(1.07,1.91)$ & \\
\hline & $\begin{array}{l}\text { Moderate to severe liver disease/Liver } \\
\text { failure }\end{array}$ & $1.89(1.14,3.14)$ & & $1.53(1.30,1.81)$ \\
\hline & Pancreatic insufficiency & & & $2.84(1.78,4.54)$ \\
\hline Infection & HIVIAIDS & $3.59(0.95,13.61)$ & $1.89(0.22,16.13)$ & \\
\hline \multirow[t]{2}{*}{ Respiratory } & Chronic airways disease & $1.68(1.5 \mathrm{I}, 1.86)$ & $1.60(1.44,1.77)$ & $\mathrm{I} .4 \mathrm{I}(\mathrm{I} .27, \mathrm{I} .56)$ \\
\hline & Pulmonary circulation disorders & & $0.95(0.76,1.18)$ & \\
\hline \multirow[t]{6}{*}{ Neurology } & Dementia & $2.15(1.94,2.37)$ & & $1.71(1.52,1.93)$ \\
\hline & Epilepsy & & & $1.03(0.89,1.19)$ \\
\hline & Migraine & & & $0.45(0.14,1.44)$ \\
\hline & Other neurological disorders & & $1.20(1.02,1.40)$ & \\
\hline & Paralysis or Hemiplegia/paraplegia & $0.65(0.20,2.10)$ & $0.80(0.34,1.86)$ & \\
\hline & Parkinson's disease & & & $1.00(0.83,1.20)$ \\
\hline \multirow[t]{4}{*}{ Psychiatry } & Anxiety & & & $0.99(0.87,1.13)$ \\
\hline & Bipolar disorder & & & $1.14(0.61,2.12)$ \\
\hline & Depression & & $0.99(0.84,1.16)$ & $1.17(1.08,1.28)$ \\
\hline & Psychotic illness/psychosis & & $1.93(1.20,3.12)$ & $1.99(1.76,2.26)$ \\
\hline \multirow[t]{5}{*}{ Rheumatology } & Allergies & & & $1.01(0.88,1.16)$ \\
\hline & Gout & & & $1.23(1.03,1.47)$ \\
\hline & Inflammation/pain & & & $0.91(0.81,1.02)$ \\
\hline & Pain & & & $1.03(0.95,1.12)$ \\
\hline & Rheumatic disease & $0.88(0.73,1.07)$ & $0.88(0.72,1.06)$ & \\
\hline \multirow[t]{3}{*}{ Urology } & Benign prostatic hyperplasia & & & $0.86(0.73,1.00)$ \\
\hline & Incontinence & & & $0.68(0.56,0.83)$ \\
\hline & Renal disease/failure & $1.67(1.46,1.92)$ & $1.63(1.41,1.90)$ & $1.09(0.58,2.04)$ \\
\hline
\end{tabular}

tumor had the highest 1-year relative mortality with an adjusted OR of 4.33 (CI: 3.57-5.25). The full list of OR of the single components are found in Tables 3 and 4.

\section{Discussion}

This study evaluated the predictive ability for mortality, including both the discrimination and calibration of different comorbidity indices among hip fracture patients in Denmark. Good discrimination was achieved by using a base model 


\section{ROC-curves for 30 day mortality \\ Base model: age and gender \\ Scores included continuous variables (not as categories)}

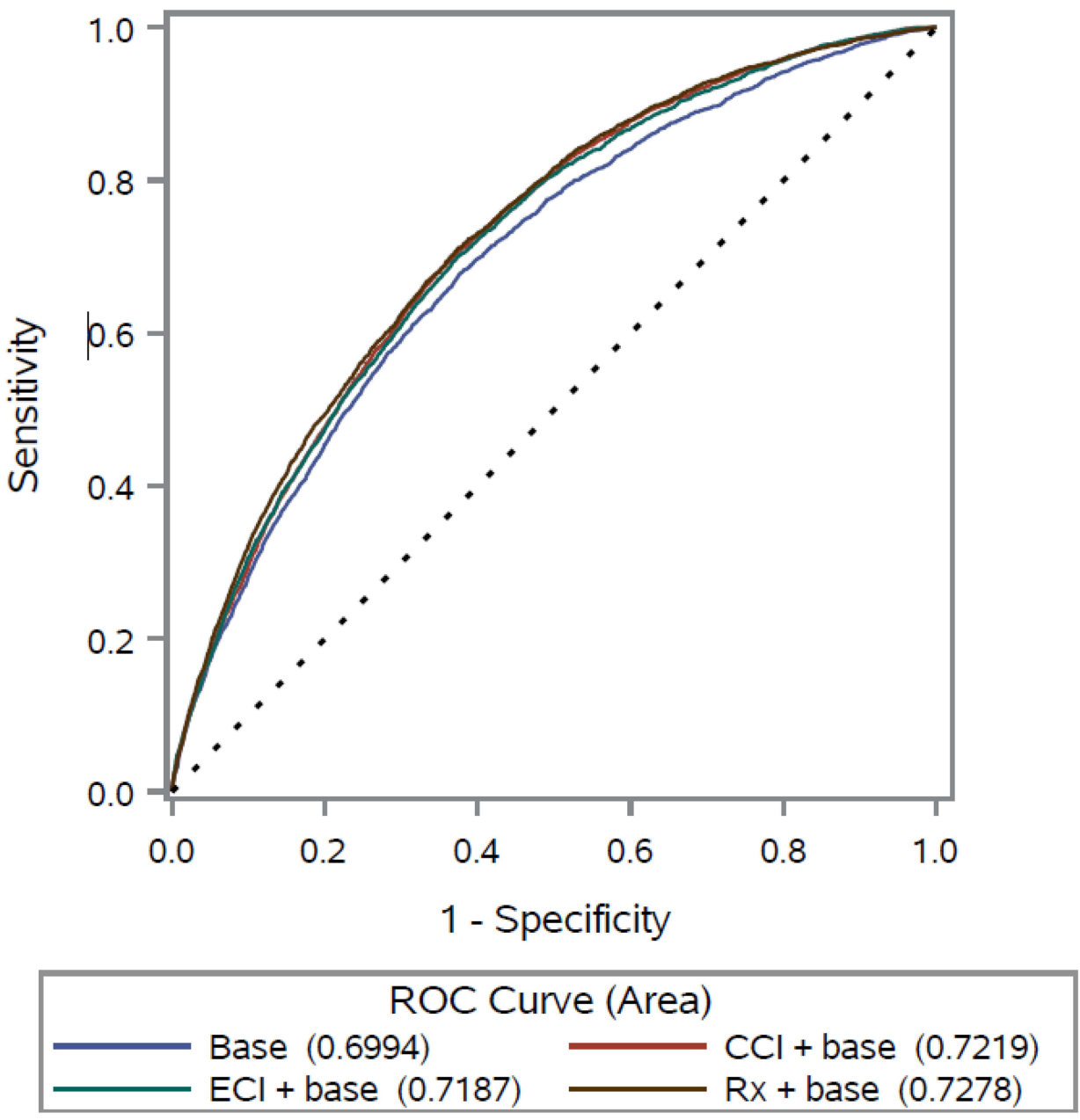

Figure I Receiver operating characteristic (ROC) curves for 30-day mortality.

(age and gender) (c-statistic was 0.70 and 0.68 for 30-day and 1-year mortality, respectively). The discrimination was improved by adding comorbidity-indices individually or in combination to the base model. The best discrimination was for CCI combined with the Rx-risk index and base model (c-statistic was 0.74 and 0.73 , for 30-day and 1-year mortality, respectively). However, the improvement was marginal. Calibration of indices was similar.

\section{Methodological Considerations}

In our study, we included both in-hospital and outpatient diagnoses reducing the potential of underreporting of specific diagnosis codes and ensuring a completeness of data. The Rx-risk index is based on pharmacy dispensings, which have good registration validation and completeness. ${ }^{18}$ However, prescribed but failed to fill-in medication was not included, which has shown to account for about $10 \%$ of general practitioners prescriptions in Denmark. ${ }^{23}$ As well as patient compliance after the dispensing is unknown. Data were collected by a variety of clinicians as part of daily routine work. Therefore, coding errors of specific diagnosis codes are unavoidable. However, coding errors will be distributed evenly in large registers, which will lead to non-differentiated misclassification. Lastly, our study was performed on a hip fracture population in Denmark, which might be different from other populations. ${ }^{24}$ Generalizing our results on other patient groups or geographical areas, should be done with caution. 


\section{ROC-curves for $\mathbf{1}$ year mortality Base model: age and gender Scores included continuous variables (not as categories)}

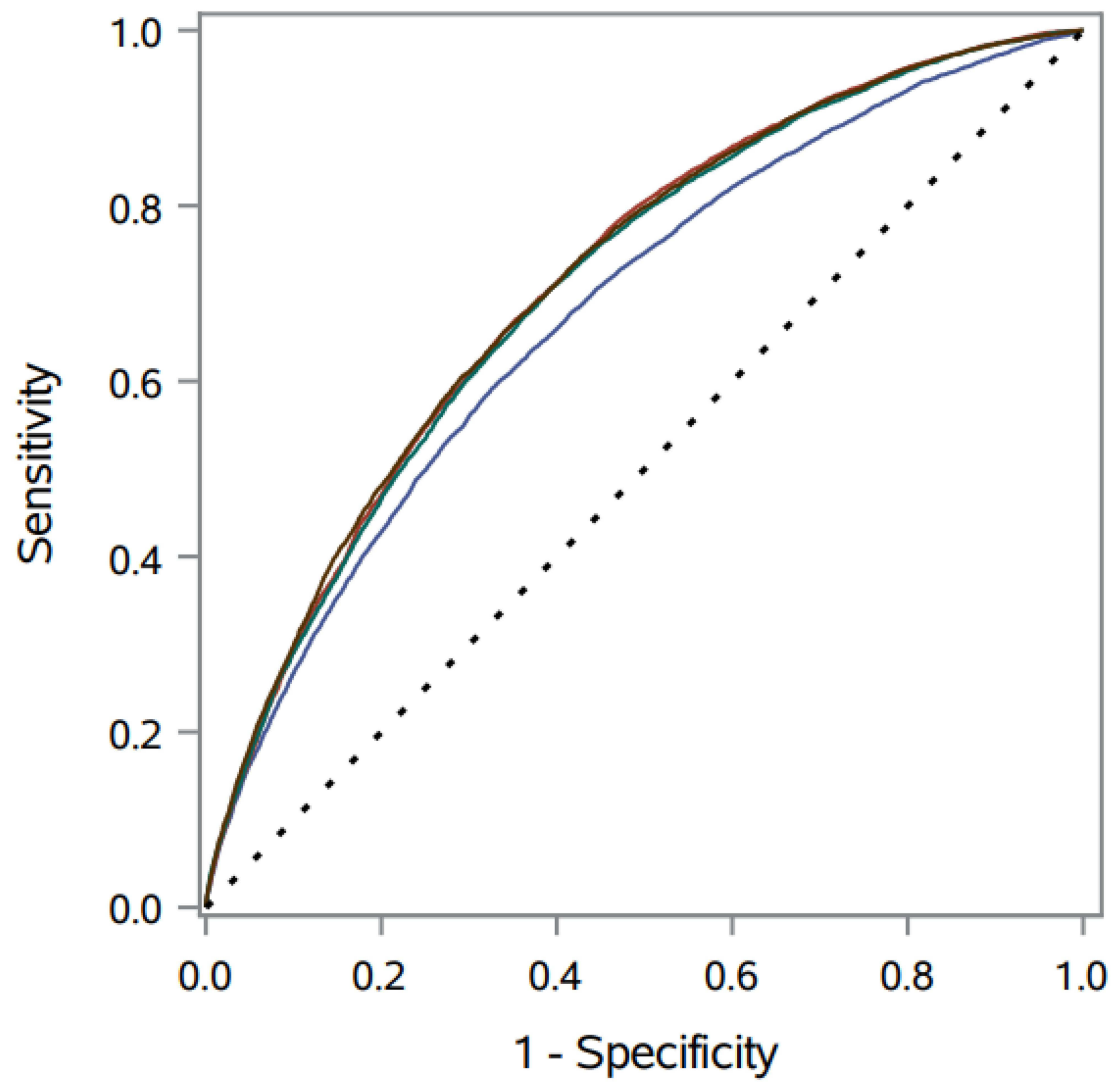

\section{ROC Curve (Area)}

Base (0.6799)

$\mathrm{CCl}+$ base $(0.7152)$

$\mathrm{ECl}+$ base $(0.7102)$

$\mathrm{Rx}+$ base $(0.7163)$

Figure 2 Receiver operating characteristic (ROC) curves for I-year mortality.

\section{Interpretation}

CCI, ECI and Rx-risk index have been compared in different settings. ${ }^{7,8,11,25-28}$ Some studies have compared indices different from ours. ${ }^{7,11,27}$ Other studies have evaluated indices in cohorts not entirely comparable to ours ${ }^{25-28}$ such as cohort of arthroplasty patients. Hip fracture patients are older, more frail and comorbid, acutely operated compared with elective arthroplasty patients, and mortality risk is substantially different in hip fracture versus arthroplasty patients. The most comparable study to ours is a Swedish study by Bülow et. al. ${ }^{8}$ They reported c-statistics for 30-day mortality of 0.67 (95\% CI: 0.67-0.68) for ECI and 0.69 (95\% CI: 0.68-0.69) for CCI, both including age and gender. These c-statistics were slightly lower than in the present study, which may be due to differences in patient characteristics, since Bülow et al included all age groups and only patients treated with arthroplasty due to hip fracture. Furthermore, Bülow et al only used diagnoses 1-year lookback period to form the comorbidity indices, while we used diagnoses 10 years prior. We used a 10-year lookback period, since this has been shown to discriminate 1-year mortality slightly better. ${ }^{29}$

Only few previous studies find a strong discrimination ability for mortality with a c-statistic $>0.8$. This may be due to that the index has either been developed for a specific context other than the investigated or developed as a "one-fits-all" 
Table 4 Odds Ratio (OR) for I-Year Mortality Among 31,443 Hip Fracture Patients. Comparing Patients with and without Specific Components of Charlson Comorbidity Index, Elixhauser Comorbidity Index and Rx-Risk Index. Adjusted for Age, Gender, and the Remaining Index Components of the Index of Interest

\begin{tabular}{|c|c|c|c|c|}
\hline \multicolumn{2}{|l|}{ Component } & \multirow{2}{*}{$\begin{array}{l}\text { Charlson Comorbidity } \\
\text { Index }\end{array}$} & \multirow{2}{*}{$\begin{array}{l}\text { Elixhauser Comorbidity } \\
\text { Index }\end{array}$} & \multirow{2}{*}{$\begin{array}{c}\text { Rx-Risk Index } \\
0.83(0.53,1.30)\end{array}$} \\
\hline Addiction & Alcohol dependency/abuse & & & \\
\hline & Drug abuse & & $1.05(0.65,1.71)$ & \\
\hline & Smoking cessation & & & $1.71(1.28,2.30)$ \\
\hline \multirow[t]{6}{*}{ Cancer } & Leukemia & $2.09(1.54,2.84)$ & & \\
\hline & Lymphoma & $1.58(1.25,1.99)$ & $1.53(1.21,1.93)$ & \\
\hline & Malignancies & & & $1.46(0.66,3.21)$ \\
\hline & Metastatic solid tumor & $4.33(3.57,5.25)$ & $4.02(3.30,4.89)$ & \\
\hline & Tumor (without metastasis) & $1.36(1.27,1.46)$ & $1.44(1.33,1.56)$ & \\
\hline & Weight loss & & $1.60(1.37,1.85)$ & \\
\hline \multirow[t]{16}{*}{ Cardiovascular } & Anemia, blood loss & & $1.16(0.88,1.54)$ & \\
\hline & Anemia, deficiency & & $1.20(1.04,1.38)$ & \\
\hline & Anticoagulants & & & $1.07(0.98,1.16)$ \\
\hline & Antiplatelets & & & $1.24(1.16,1.31)$ \\
\hline & Arrhythmia & & $1.14(1.07,1.22)$ & $1.44(1.29,1.60)$ \\
\hline & Cerebrovascular disease & $1.19(1.11,1.28)$ & & \\
\hline & Coagulopathy & & $1.05(0.80,1.37)$ & \\
\hline & Congestive heart failure & $1.53(1.40,1.68)$ & $1.38(1.26,1.52)$ & $1.50(1.39,1.63)$ \\
\hline & Fluid and electrolyte disorders & & $1.49(1.39,1.60)$ & \\
\hline & Hyperlipidemia & & & $0.77(0.73,0.83)$ \\
\hline & Hypertension & & $0.96(0.90,1.02)$ & $1.11(1.05,1.18)$ \\
\hline & Ischemic heart disease angina & & & $1.06(0.96,1.18)$ \\
\hline & Ischemic heart disease hypertension & & & $0.94(0.89,1.00)$ \\
\hline & Myocardial infarction & $1.07(0.95,1.20)$ & & \\
\hline & Peripheral vascular disease & $1.29(I .18,1.4 I)$ & $1.26(1.15,1.38)$ & \\
\hline & Valvular disease & & $1.27(1.15,1.40)$ & \\
\hline \multirow[t]{7}{*}{ Endocrinology } & Diabetes, uncomplicated & $1.17(1.07,1.29)$ & $1.13(1.01,1.25)$ & \multirow[t]{2}{*}{$1.22(1.12,1.34)$} \\
\hline & Diabetes, complicated & $1.03(0.82,1.29)$ & $1.13(0.99,1.30)$ & \\
\hline & Hyperthyroidism & & & $1.15(0.68,1.94)$ \\
\hline & Hypothyroidism & & $0.89(0.76,1.03)$ & $0.99(0.90,1.10)$ \\
\hline & Obesity & & $0.93(0.77,1.12)$ & \\
\hline & Osteoporosis/Paget's & & & $0.84(0.77,0.91)$ \\
\hline & Steroid-responsive disease & & & \\
\hline
\end{tabular}


Table 4 (Continued).

\begin{tabular}{|c|c|c|c|c|}
\hline \multicolumn{2}{|l|}{ Component } & \multirow{2}{*}{$\begin{array}{l}\text { Charlson Comorbidity } \\
\text { Index }\end{array}$} & \multirow{2}{*}{$\begin{array}{l}\text { Elixhauser Comorbidity } \\
\text { Index } \\
1.01(0.83,1.23)\end{array}$} & \multirow{2}{*}{$\begin{array}{l}\text { Rx-Risk Index } \\
1.20(1.13,1.27)\end{array}$} \\
\hline Gastrointestinal & $\begin{array}{l}\text { Gastroesophageal reflux disease/peptic } \\
\text { ulcer }\end{array}$ & & & \\
\hline & Irritable bowel syndrome & & & $1.09(0.86,1.38)$ \\
\hline & Mild liver disease & $1.60(1.24,2.06)$ & $1.27(1.03,1.56)$ & \\
\hline & $\begin{array}{l}\text { Moderate to severe liver disease/Liver } \\
\text { failure }\end{array}$ & I.7I $(1.17,2.49)$ & & $1.65(1.45,1.88)$ \\
\hline & Pancreatic insufficiency & & & $2.84(1.99,4.07)$ \\
\hline Infection & HIVIAIDS & I.5I $(0.45,5.07)$ & $1.35(0.25,7.23)$ & \\
\hline \multirow[t]{2}{*}{ Respiratory } & Chronic airways disease & $1.60(1.48,1.73)$ & $1.50(1.39,1.62)$ & $\mathrm{I} .3 \mathrm{I}(\mathrm{I} .22, \mathrm{I} .4 \mathrm{I})$ \\
\hline & Pulmonary circulation disorders & & $1.08(0.92,1.26)$ & \\
\hline \multirow[t]{6}{*}{ Neurology } & Dementia & $2.39(2.21,2.58)$ & & $1.82(1.66,1.99)$ \\
\hline & Epilepsy & & & $0.95(0.86,1.05)$ \\
\hline & Migraine & & & $0.47(0.24,0.90)$ \\
\hline & Other neurological disorders & & $1.28(1.15,1.43)$ & \\
\hline & Paralysis or Hemiplegia/paraplegia & $0.72(0.37,1.39)$ & $0.87(0.52,1.45)$ & \\
\hline & Parkinson's disease & & & $1.03(0.91,1.16)$ \\
\hline \multirow[t]{4}{*}{ Psychiatry } & Anxiety & & & $1.12(1.02,1.22)$ \\
\hline & Bipolar disorder & & & $0.87(0.55,1.37)$ \\
\hline & Depression & & $1.05(0.94,1.17)$ & $1.25(1.18,1.33)$ \\
\hline & Psychotic illness/psychosis & & $1.73(1.22,2.45)$ & $1.87(1.70,2.06)$ \\
\hline \multirow[t]{5}{*}{ Rheumatology } & Allergies & & & $0.91(0.83,1.00)$ \\
\hline & Gout & & & $1.18(1.03,1.35)$ \\
\hline & Inflammation/pain & & & $0.88(0.8 \mathrm{I}, 0.95)$ \\
\hline & Pain & & & $1.24(1.17,1.32)$ \\
\hline & Rheumatic disease & $0.97(0.85,1.09)$ & $0.94(0.83,1.07$ & \\
\hline \multirow[t]{3}{*}{ Urology } & Benign prostatic hyperplasia & & & $0.93(0.82,1.04)$ \\
\hline & Incontinence & & & $0.69(0.60,0.78)$ \\
\hline & Renal disease/failure & $1.60(1.44,1.79)$ & $1.60(1.42,1.81)$ & $1.62(1.02,2.56)$ \\
\hline
\end{tabular}

index. Although, a perfect comorbidity index to predict mortality is difficult to obtain since other complex factors affects mortality ${ }^{30,31}$ it is important to identify the index with the best c-statistic in order to control for confounding and decrease bias due to comorbidity in epidemiological studies as much as possible. ${ }^{32}$

Our study findings regarding the single components of the different indices may improve our knowledge towards generating a new comorbidity index with a strong prediction ability for mortality among hip fracture patients. First, we found a series of components which had a protective effect on mortality, such as migraine, rheumatic disease and para- $/$ 
hemiplegia. This may be contributed to surveillance bias, since patients with these diseases will be followed closer by health professionals and therefore have a lower threshold for identifying and treating other milder cases of comorbidities. Secondly, we found that the weighting of some components was in discrepancy with the OR for mortality when seeing the single component as an exposure for mortality. For example, dementia in CCI is weighted as 1, even though dementia has an OR more a like the components weighted with 2 or 6 . The discrepancy in the weighting and the OR for mortality may happen due to more effective treatment available today, which would lower mortality compared to 1987 when the CCI was developed. Lastly, we found a series of components which increased the OR for death. Most of which is well known to increase mortality in a geriatric hip fracture population, such as cancer, ${ }^{33-35}$ cardiovascular disease, ${ }^{33-35}$ dementia, ${ }^{33-35}$ chronic airways disease ${ }^{33-35}$ as well as liver- and renal diseases. ${ }^{33-35}$

To summarize, we found the highest 30-day- and 1-year mortality discrimination ability when combining CCI and Rx-risk with age and gender (c-statistic $=0.74$ and $c$-statistic $=0.73$, respectively). However, age and gender alone had almost the same discrimination ability (c-statistic $=0.70, \mathrm{CI}$ : $0.69-0.71$ and c-statistic $=0.68, \mathrm{CI}$ : $0.67-0.69$, respectively) as complex combined indices. Our findings indicate that 1) it will be possible to develop a new comorbidity index, combining data on both diagnosis and pharmacy dispensing specifically for hip fracture patients, 2) the need for investigating weighting of the components in a hip fracture population, as they might be different from original comorbidity indices weightings. At last, hip fracture patients are often elderly and frail and adjusting for complex comorbidity index will not remove all confounding since other factors are associated with mortality. The inclusion of other domains such as level of function (i.e activities of daily living), cognition, social support, and nutrition would likely further improve prediction ability of comorbidity indices and controlling for confounding. ${ }^{30,31}$

\section{Disclosure}

The authors report no conflicts of interest in this work. The authors' affiliation, Department of Clinical Epidemiology is, however, involved in studies with funding from various companies as research grants to (and administered by) Aarhus University.

\section{References}

1. Pedersen AB, Ehrenstein V, Szépligeti SK, et al. Thirty-five - year trends in first-time hospitalization for hip fracture, 1-year mortality, and the prognostic impact of comorbidity: a Danish Nationwide Cohort Study. Epidemiology. 2017;28:898-905. doi:10.1097/EDE.0000000000000729

2. Lawrence VA, Hilsenbeck SG, Noveck H, Poses RM, Carson JL. Medical complications and outcomes after hip fracture repair. Arch Intern Med. 2002;162:2053-2057. doi:10.1001/archinte.162.18.2053

3. Beringer TRO. Mortality and morbidity after hip fractures. Br Med J. 1994;308:343. doi:10.1136/bmj.308.6924.343b

4. Cher EWL, Allen JC, Sen HT, Koh JSB. Comorbidity as the dominant predictor of mortality after hip fracture surgeries. Osteoporos Int. 2019;30:2477-2483. doi:10.1007/s00198-019-05139-8

5. Charlson ME, Pompei P, Ales KL. A new method of classifying prognostic comorbidity in longitudinal studies: development and validation. J Chronic Dis. 1987;40:373-383. doi:10.1016/0021-9681(87)90171-8

6. Vesterager JD, Kristensen PK, Petersen I, Pedersen AB. Hospital variation in the risk of infection after hip fracture surgery: a population-based cohort study including 29,598 patients from 2012-2017. Acta Orthop. 2020;92:215-221. doi:10.1080/17453674.2020.1863688

7. Ondeck NT, Bovonratwet P, Ibe IK, et al. Discriminative ability for adverse outcomes after surgical management of hip fractures: a comparison of the Charlson comorbidity index, elixhauser comorbidity measure, and modified frailty index. J Orthop Trauma. 2018;32:231-237. doi:10.1097/ BOT.0000000000001140

8. Bülow E, Cnudde P, Rogmark C, Rolfson O, Nemes S. Low predictive power of comorbidity indices identified for mortality after acute arthroplasty surgery undertaken for femoral neck fracture. Bone $J t J$. 2019;101B:104-112. doi:10.1302/0301-620X.101B1.BJJ-2018-0894.R1

9. Elixhauser A, Steiner C, Harris DR. Comorbidity measures for use with administrative data. Med Care. 1998;36:8-27. doi:10.1097/00005650199801000-00004

10. Bass E, French DD, Bradham DD, Rubenstein LZ. Risk-adjusted mortality rates of elderly veterans with hip fractures. Ann Epidemiol. 2007;17:514-519. doi:10.1016/j.annepidem.2006.12.004

11. Lu CY, Barratt J, Vitry A, Roughead E. Charlson and Rx-Risk comorbidity indices were predictive of mortality in the Australian health care setting. J Clin Epidemiol. 2011;64:223-228. doi:10.1016/j.jclinepi.2010.02.015

12. Johnson ML, El-Serag HB, Tran TT, Hartman C, Richardson P, Abraham NS. Adapting the Rx-Risk-V for mortality prediction in outpatient populations. Med Care. 2006;44:793-797. doi:10.1097/01.mlr.0000218804.41758.ef

13. Kristensen PK, Röck ND, Christensen HC. The Danish multidisciplinary hip fracture registry 13-year results from a population-based cohort of hip fracture patients. Clin Epidemiol. 2020;2020:9-21.

14. Steyerberg EW, Vickers AJ, Cook NR, et al. Assessing the performance of prediction models: a framework for some traditional and novel measures. Epidemiology. 2013;21:128-138. doi:10.1097/EDE.0b013e3181c30fb2

15. Schmidt M, Schmidt SAJ, Adelborg K, et al. The Danish health care system and epidemiological research: from health care contacts to database records. Clin Epidemiol. 2019;2019:563-591. doi:10.2147/CLEP.S179083 
16. Schmidt M, Schmidt SA, Sandegaard JL, Ehrenstein V, Pedersen L. The Danish national patient registry: a review of content, data quality, and research potential. Clin Epidemiol. 2015;2015:449-490. doi:10.2147/CLEP.S91125

17. Pratt NL, Kerr M, Barratt JD, et al. The validity of the Rx-Risk comorbidity index using Medicines mapped to the Anatomical Therapeutic Chemical (ATC) classification system. BMJ Open. 2018;8:1-8. doi:10.1136/bmjopen-2017-021122

18. Wallach H, Sørensen H, Hallas J, Sørensen HT, Hallas J. The Danish national prescription registry. Scand J Public Health. 2011;39:38-41. doi: $10.1177 / 1403494810394717$

19. Hanley A, Mcneil J, Ph D. The meaning and use of the area under a receiver operating characteristic (ROC) curve. Radiology. 1982;143:29-36. doi:10.1148/radiology.143.1.7063747

20. Heagerty PJ, Zheng Y, Biometrics S, Mar N, Heagerty PJ. Survival model predictive accuracy and ROC curves published by: international biometric society stable. Biometrics. 2021;61:92-105.

21. Vandenbroucke JP, Von EE, Altman DG, et al. Strengthening the reporting of observational studies in epidemiology (STROBE): explanation and elaboration. PLoS Med. 2007;4:e297. doi:10.1371/journal.pmed.0040297

22. Ludvigsson JF, Håberg SE, Knudsen GP, Lafolie P, Zoega H. Ethical aspects of registry-based research in the Nordic countries. Clin Epidemiol. 2015;7:491-508. doi:10.2147/CLEP.S90589

23. Pottegård A, Christensen R, Houji A. Primary non-adherence in general practice: a Danish register study. Eur J Clin Pharmacol. 2014;70:757-763. doi:10.1007/s00228-014-1677-y

24. Kanis JA, Odén A, McCloskey EV, Johansson H, Wahl DA, Cooper C. A systematic review of hip fracture incidence and probability of fracture worldwide. Osteoporos Int. 2012;23:2239-2256. doi:10.1007/s00198-012-1964-3

25. Inacio MCS, Pratt NL, Roughead EE, Graves SE. Using medications for prediction of revision after total joint arthroplasty. $J$ Arthroplasty. 2015;30:2061-2070. doi:10.1016/j.arth.2015.06.009

26. Inacio MCS, Pratt NL, Roughead EE, Graves SE. Evaluation of three co-morbidity measures to predict mortality in patients undergoing total joint arthroplasty. Osteoarthr Cartil. 2016;24:1718-1726. doi:10.1016/j.joca.2016.05.006

27. Cai M, Liu E, Zhang R, et al. Comparing the performance of Charlson and elixhauser comorbidity indices to predict in-hospital mortality among a Chinese population. Clin Epidemiol. 2020;12:307-316. doi:10.2147/CLEP.S241610

28. Li B, Evans D, Faris P, Dean S, Quan H. Risk adjustment performance of Charlson and Elixhauser comorbidities in ICD-9 and ICD-10 administrative databases. BMC Health Serv Res. 2008;8:1-7. doi:10.1186/1472-6963-8-12

29. Madsen M, Ehrenstein V, Sørensen HT. Length of comorbidity lookback period and predicting one-year mortality based on registry data from Denmark (abstract). Pharmacoepidemiol Drug Saf. 2015;24:392-393.

30. Kimura A, Matsumoto Y, Wakata Y, Oyamada A, Ohishi M, Fujiwara T. Predictive factors of mortality of patients with fragility hip fractures at 1 year after discharge: a multicenter, retrospective study in the northern Kyushu district of Japan. J Orthopaedic Surg. 2019;27:1-8.

31. Smith T, Pelpola K, Ball M, Ong A, Myint PK. Pre-operative indicators for mortality following hip fracture surgery: a systematic review and meta-analysis. Age Ageing. 2014;43:464-471. doi:10.1093/ageing/afu065

32. Schneeweiss S, Seeger JD, Maclure M, Wang PS, Avorn J, Robert J. Performance of comorbidity scores to control for confounding in epidemiologic studies using claims data. Am J Epidemiol. 2001;154:854-864. doi:10.1093/aje/154.9.854

33. Roche JJW, Wenn RT, Sahota O, Moran CG. Effect of comorbidities and postoperative complications on mortality study. BMJ. 2005;55:1-5.

34. Guzon-illescas O, Fernandez EP, Villarias NC, et al. Mortality after osteoporotic hip fracture: incidence, trends, and associated factors. $J$ Orthop Surg Res. 2019;6:1-9.

35. Hu F, Jiang C, Shen J, Tang P, Wang Y. Preoperative predictors for mortality following hip fracture surgery: a systematic review and meta-analysis. Injury. 2012;43:676-685. doi:10.1016/j.injury.2011.05.017

Clinical Epidemiology

Dovepress

\section{Publish your work in this journal}

Clinical Epidemiology is an international, peer-reviewed, open access, online journal focusing on disease and drug epidemiology, identification of risk factors and screening procedures to develop optimal preventative initiatives and programs. Specific topics include: diagnosis, prognosis, treatment, screening, prevention, risk factor modification, systematic reviews, risk \& safety of medical interventions, epidemiology \& biostatistical methods, and evaluation of guidelines, translational medicine, health policies \& economic evaluations. The manuscript management system is completely online and includes a very quick and fair peer-review system, which is all easy to use.

Submit your manuscript here: https://www.dovepress.com/clinical-epidemiology-journal 